\title{
Panorama de la salud-enfermedad física y mental de la comunidad de Daxthá
}

\author{
Panorama of the Physical and Mental Health-illness of the Community of Daxthá
}

\author{
Fernando Bolaños ${ }^{a}$ y Jorge A. Guzmán Cortés ${ }^{b}$
}

\begin{abstract}
:
The objective of this work is to show an overview of the main physical and mental health problems in the Dhaxtá community, in the municipality of Actopan, Hidalgo State, Mexico. An analysis of morbidity and mortality data is carried out at the national, state and municipal levels. Based on the problems indicated by the statistics, some strategic lines of attention to physical and mental health are considered from a health promotion approach with a gender perspective, aimed at laying the foundations for the implementation of actions to benefit the health of the community.
\end{abstract}

Keywords:

community health, mental health, health promotion, gender, care

Resumen:

Este trabajo tiene como objetivo mostrar un panorama general de las principales problemáticas de salud física y mental en la comunidad de el Dhaxtá, en el municipio de Actopan del Estado de Hidalgo, México. Se realiza un análisis de los datos de morbilidad y mortalidad a nivel nacional, estatal y municipal. Con base en la problemática señalada por las estadísticas, se consideran algunas líneas estratégicas de atención a la salud física y mental desde un enfoque de promoción de la salud con perspectiva de género, dirigidas a sentar las bases para la implementación de acciones en beneficio de la salud de la comunidad.

Palabras Clave:

salud comunitaria, salud mental, promoción de la salud, género, atención

\section{Introducción}

La comunidad de Daxthá, se encuentra en el municipio de Actopan en el estado de Hidalgo, en una región geográfica denominada Valle del Mezquital, en la región oriente de México. Con base en la labor de promoción de la salud que impulsa la Universidad Autónoma del Estado de Hidalgo -UAEH- en toda la región, en la Escuela Superior de Actopan de la UAEH, ubicada dentro de esta misma comunidad, se impulsa actualmente un proyecto más amplio de promoción de la salud dirigido por profesores investigadores de la licenciatura en psicología. El objetivo consiste en recuperar las experiencias y acciones de salud mental realizadas en la labor universitaria en Daxthá durante los años recientes, e impulsar una iniciativa mayor y a más largo plazo que articule a los actores públicos y civiles de la comunidad, e incorpore la recopilación de datos desde una lógica de investigación-acción. Este trabajo forma parte de ese esfuerzo de promoción de la salud.

\section{La Salud en México}

La Organización Mundial de la Salud considera a la salud como un estado de completo bienestar físico, mental y social, y no solamente la ausencia de afecciones o enfermedades (OMS, 2015). Aunque tradicionalmente solo se toma en cuenta la salud física, también es importante destacar que este bienestar abarca la Salud Mental. En ese sentido, la salud mental es un estado de bienestar, en el cual el individuo es consciente

\footnotetext{
Autor de Correspondencia, Universidad Autónoma del Estado de Hidalgo, Escuela Superior de Actopan, Email: fernando_bolanos@uaeh.edu.mx

Universidad Autónoma del Estado de Hidalgo, Escuela Superior de Actopan, ORCID: 0000-0001-7899-2183, Email: 
de sus propias capacidades, puede afrontar las tensiones normales de la vida, puede trabajar de forma productiva y fructífera, y es capaz de hacer una contribución a su comunidad; además, dentro de este concepto se incluye la prevención de trastornos mentales, así como el tratamiento y rehabilitación de las personas afectadas por dichos trastornos, pero principalmente, con la promoción del bienestar (OMS, 2015).

\section{Principales Causas de Morbilidad en México ${ }^{1}$}

Según el Informe sobre la salud de los mexicanos 2015 (SIDSS, 2015), las principales diez causas de enfermedad diferenciadas por sexo, tal y como las reporta el Sistema Nacional de Vigilancia Epidemiológica (SINAVE) ${ }^{2}$ en 2014, son las que se muestran en la tabla 1

Los datos del Informe indican, que se reportaron poco más de 46 millones de casos de enfermedad, 27 en mujeres y 19 en hombres. La causa más frecuente de atención entre los hombres fueron las reportadas como "infecciones respiratorias agudas, con 12.1 millones de casos, más de $60 \%$ del total. El segundo sitio lo ocuparon las "infecciones intestinales por otros organismos y las mal definidas", con 2.27 millones de casos. Lo anterior denota que, a diferencia de lo que ocurre en el análisis de mortalidad, la atención médica en las unidades está fuertemente dirigida hacia la atención de infecciones comunes. De hecho, la tercera causa en los hombres son las infecciones de vías urinarias, con lo que el conglomerado de estas tres categorías suma más de quince millones de registros, aproximadamente $80 \%$ del total registrado en hombres (SIDSS, 2015).

Para las mujeres la situación es similar, pues las primeras seis causas de enfermedad son las mismas que para los hombres, aunque el número absoluto es mayor. Las principales diferencias se presentan por la aparición de padecimientos propios de este sexo, como la vulvo-vaginitis aguda y la candidiasis urogenital. La octava causa de enfermedad en ambos sexos fue la hipertensión arterial. Además, la novena causa en hombres fue la diabetes, que en el caso de las mujeres ocupó la posición 12, detrás de la obesidad. Esta última tuvo el lugar 14 en los

1 Corresponden a las enfermedades o categorías diagnósticas más frecuentemente registradas en el Sistema Nacional de Vigilancia Epidemiológica en 2014. Informe sobre la salud de los mexicanos, 2015. Subsecretaría de Integración y Desarrollo del Sector Salud Dirección General de Evaluación del Desempeño. Gobierno de México. hombres. Debido a lo anterior, la hipertensión arterial es la séptima causa de enfermedad a nivel nacional, la diabetes mellitus la novena y la obesidad la décima. Es importante mencionar que la obesidad apareció por primera vez en 2014 entre las primeras 20 causas de enfermedad registradas. Se enfatiza la aparición de estas causas de atención debido a que no suelen incluirse entre las prioridades en materia de prevención (SIDSS, 2015). Como se observa en ambos casos, las enfermedades infectocontagiosas $-y$ las enfermedades asociadas a la desnutrición- siguen reportando una alta prevalencia en México, mostrando que la transición epidemiológica a enfermedades crónico-degenerativas del mundo occidental presenta un patrón más complejo para países como el nuestro, considerado por algunos como de "estancamiento y polarización" (Arredondo, Carranza, Vázquez, y Rodríguez, 2003).

Es a través de estos datos que podemos darnos cuenta que las principales causas del perfil epidemiológico nacional se pueden asociar primeramente a loque se conoce como los estilos de vida (Guerrero y León, 2010), ya que se encuentra relacionadas con la mala alimentación, el sedentarismo, entre otros hábitos; así, la obesidad, la diabetes mellitus, la hipertensión, las ulceras, las gastritis y otros padecimientos se asocian a estilos de vida particulares. Desde un enfoque de determinantes sociales de la salud, enfermedad y atención (López, Blanco, Garduño, Granados, Jarillo, López, Rivera y Tetelboin, 2010), muchas de estas causas de patologías estilos de vida-, responderían a su vez a otras causas mayores provenientes de dimensiones sociales más amplias y con carácter histórico, tales como: la pobreza, el bajo ingreso, las altas tasas de desempleo, la calidad de vida, la discriminación, entre otras determinantes, y por lo tanto, la enfermedad se consideraría como una última manifestación de estos procesos socialespersonales-biológicos (López y Blanco, 2003), y no sólo como el producto de una decisión personal o la falta de educación en salud. Entre grupos de

${ }^{2}$ Es importante considerar que las cifras de morbilidad que se derivan del SINAVE no deben considerarse como tasas de incidencia, toda vez que no representan, por diferentes razones, a todos los casos nuevos de una enfermedad. Lo que se presenta aquí, está basado en el número de casos registrados en dicho sistema. 
edad, las principales enfermedades son muy parecidas al total de la población -tabla 2-.

Se observa, que se mantienen las infecciones respiratorias agudas como principal causa de morbilidad y las infecciones intestinales como segunda causa, además de que las infecciones de vías urinarias están entre las primeras cinco. A partir de los 15 años, se presentan las úlceras, gastritis y duodenitis; a partir de los 50 años, la hipertensión arterial se presenta como la quinta causa de atención, seguida de la diabetes mellitus (SIDSS, 2015).

A pesar de que las principales causas son muy parecidas entre los diferentes grupos de edad, su importancia relativa es diferente: las infecciones respiratorias agudas representaron el $81 \%$ de las enfermedades en los menores de un año, porcentaje que decrece con la edad hasta el $46 \%$ en los adultos de 65 años o más. Por otra parte, las infecciones intestinales se mantienen a lo largo de los grupos de edad, muy cerca del $10 \%$ del total de las enfermedades (SIDSS, 2015).

\section{Principales causas de muerte en México}

Para el 2015 se registraron 655688 defunciones, y la esperanza de vida de la población es de 75.3 años para el año 2017. Las defunciones que tienen un mayor peso relativo, se agrupan en las enfermedades del sistema circulatorio -25.5\%-; enfermedades endocrinas, nutricionales $y$ metabólicas -17.5\%-, y; los tumores malignos $13 \%$ - Al interior de estas agrupaciones, hay una diversidad de enfermedades que de manera desagregada dan un mejor contexto epidemiológico por el cual fallece la población. Si se considera este desglose, entonces las primeras siete causas de muerte corresponden a la diabetes mellitus $-15 \%$; las enfermedades isquémicas del corazón -13.4\%-; las del hígado $5.4 \%$-; las cerebrovasculares -5.2\%-; las crónicas de las vías respiratorias inferiores $-4 \%-$, las hipertensivas $-3.5 \%-$, y; las agresiones -3.2\%-. En conjunto, estas enfermedades representan casi la mitad -49.7\%- de los decesos totales (INEGI, 2017a).

Cuando se revisan estos datos por grupo de edad, se pueden observar la variabilidad de las causas de mortalidad en general, y específicas según el sexo; es decir, según se trate de las causas de muerte de menores de un año de edad, niños de 1 a 14 años, jóvenes de 15 a 29 años, adultos de 30 a 59 años o en la población de 60 y más años, se presentarán variaciones y diferenciales significativos según la edad y el sexo.

Por ejemplo, un patrón claramente diferenciado por sexo que abarca a varios grupos de edad, son las muertes violentas de los varones, la cuales incluyen las denominadas causas externas de morbilidad y mortalidad -agresiones, accidentes de transporte y lesiones autoinflingidas intencionalmente-. En suma, estas tres causas pueden llegar a representar la segunda o tal vez la primera causa de muerte de los varones mexicanos de entre 1 a 14 años, 15 a 29 y adultos de 30 a 59 años. Estos datos reflejan la sobremortalidad masculina (INEGI, 2017a) producto de las ideas, roles y prácticas dominantes de género para los varones (Scott, 1990; Connell, 2003; de Keijzer, 2014) como una determinante social de la salud, de igual forma que los altos índices de muerte de mujeres en el proceso de embarazo, parto y puerperio responderían a causas sociales prevenibles.

Por lo tanto, otras causas de fallecimiento emergentes a atender son la mortalidad materna (INEGI, 2017a), de la cual se ha planteado la reducción de la tasa a menos de 70 por cada 100 mil nacidos vivos para el año 2030. En México la razón de mortalidad materna se encuentra por debajo de este nivel -en 2015 es de 34.6 defunciones por cada 100 mil nacidos vivos-. Estas muertes son evitables y la mayoría ocurre en la población de mayor rezago social. Otra causa emergente en México es la mortalidad por Virus de la Inmunodeficiencia Humana -VIH-, cuya tasa para 2015 es de 3.9 defunciones por cada 100 mil habitantes, siendo Tabasco - 10.4-, Quintana Roo -9.5-, Campeche -8.7-, Veracruz 8.7- y Colima -8- las entidades con mayor mortalidad por esta causa. Se destaca que, si bien no hay cura, "el tratamiento con antirretrovirales eficaces permite mantener controlado el virus..., haciendo que la persona infectada lleve una vida saludable, larga y productiva". También se pone atención en las defunciones por tumores malignos, ya que en 2015 fallecieron 85,201 personas por esta causa. En los hombres, las defunciones por tumores de próstata -15.4\%-y los tumores de la tráquea, de los bronquios y del pulmón -10.4\%- son las dos principales causas de muerte en este grupo de defunciones. Sobre esta última, la OMS argumenta que "alrededor del $70 \%$ de la carga de cáncer de pulmón puede achacarse 
al tabaquismo como única causa". El consumo del alcohol también es "un factor de riesgo para muchos tipos de cáncer como los de boca, faringe, laringe, esófago, hígado, colon y recto, además de mama". En las mujeres, el tumor maligno de mama $-14.4 \%$ - y del cuello del útero -9.2\%- figuran entre las principales causas de muerte por tumores. En el tumor de mama, los principales factores de riesgo se vinculan a "una exposición prolongada a estrógenos endógenos, una menarquía precoz, una menopausia tardía y una edad madura cuando se presenta el primer parto. Cabe señalar, que en todos los tumores la prevención y el diagnóstico oportuno son la mejor forma de detectar y dar tratamiento oportuno a esta enfermedad. Finalmente, otra causa de fallecimiento emergente a atender en México es la desnutrición, ya que, en 2015, se registraron 7, 037 defunciones por esta causa: $48.6 \%$ eran hombres y $51.4 \%$ mujeres; por edad, $81.1 \%$ tenían 60 y más años de edad.

\section{Determinantes sociales de la salud}

Además de los datos sobre morbilidad y mortalidad en México, es necesario considerar a otros elementos significativos del contexto social, económico y político del país, ya que, en muchos casos, estos procesos suelen convertirse en determinantes sociales de la salud, tal es el caso de la pobreza ysu afectación a la calidad de vida de las personas y comunidades. Por ejemplo, los datos señalados por el Consejo Nacional de Evaluación de la Política de Desarrollo Social (CONEVAL, 2012), miden la pobreza desde un acercamiento multidimensional incluyendo al ingreso corriente per cápita, el rezago educativo promedio en el hogar, acceso a los servicios de salud, acceso a la seguridad social, la calidad y espacios de la vivienda, el acceso a los servicios básicos en la vivienda, el acceso a la alimentación, y el grado de cohesión social. De esta forma, los datos sobre estas condiciones de vida que afectan la salud-enfermedad-atención, señalan que para 2012 existían a nivel nacional 53 millones, 349.9 mil personas en situación de pobreza -45.5\%- y 11 millones, 529 mil en pobreza extrema -9.8\%-. Ello implica, que aproximadamente el $55.3 \%$ de la población nacional vivía en 2012 en condiciones de pobreza o extrema pobreza. Esta misma medición multidimensional, reportó que para 2014 el porcentaje nacional es de $46.2 \%$ de pobres -55.3 millones- y 9.5 -11.4 millones- para la pobreza extrema; es decir, que la pobreza aumentó en casi un punto porcentual y la pobreza extrema bajó sólo tres décimas (CONEVAL, 2015). Ella muestra una tendencia de aumento en el empobrecimiento de la población mexicana que actualmente es de $55.7 \%-66.7$ millones- del total, según los datos oficiales, variable que es importante a considerar en sus efectos sobre la salud de la población, y más aún, en los obstáculos para la prevención y atención de la salud física y mental de todos los grupos de edad.

\section{La salud mental para México}

Además de las tendencias de la enfermedad y muerte en México, para comprender el panorama epidemiológico de la salud en la comunidad de Dhaxtá, es necesario conocer los principales datos en materia de salud mental a nivel nacional y estatal. La salud mental es un aspecto importante de la salud integral, inseparable del resto y se refiere no solamente a la ausencia de enfermedades y trastornos mentales, sino también al ejercicio de las potencialidades para la vida personal y la interacción social, que son inherentes a la naturaleza del hombre y condicionan su bienestar. Una actitud mental equilibrada permite afrontar de manera más eficaz el estrés de la vida cotidiana, realizar un trabajo fructífero y hacer aportaciones positivas a la comunidad (SSA, 2002).

La salud mental, se trata de un fenómeno complejo determinado por múltiples componentes biológicos y psicosociales. Para comprenderlo es necesario analizar las transiciones sociodemográficas, económicas, culturales, tecnológicas y políticas por las que está atravesando el país; éstas generan una importante demanda de atención de parte de las instituciones públicas, privadas y sociales debido al incremento de los trastornos mentales en la población, lo que representa mayores requerimientos de servicios de salud, así como de un profundo conocimiento de la incidencia, prevalencia y características de los padecimientos mentales y de las alternativas de atención (SSA, 2002).

Esas necesidades son mayores en la medida en que se presentan situaciones de vida más adversas -pobreza, rezago social, entre otras-. El Programa Nacional de Salud -2012-2018-, 
contempla la disminución de las adicciones al tabaco, alcohol y drogas y la atención a los problemas de salud mental respectivamente. La relevancia de la problemática de los padecimientos mentales estriba en que se estima, que por lo menos una quinta parte de la población mexicana padece en el curso de su vida algún trastorno mental: cuatro millones de adultos presentan depresión; medio millón padece esquizofrenia, un millón de personas tiene epilepsia y el $10 \%$ de los mayores de 65 años padecen algún tipo de demencia; en una tendencia creciente de estos padecimientos, en los próximos diez años la demanda de servicios de salud mental en México constituirá una de las principales presiones para el sistema de salud. Entre las quince principales causas de pérdida de vida saludable en México, se encuentran las enfermedades psiquiátricas y neurológicas; seis de ellas están relacionadas de alguna manera con trastornos mentales y con las adicciones (IESMOMS, 2011).

Al igual que en temas de salud física, la prevalencia de enfermedades mentales también tiene un diferencial por género. Los hombres presentan prevalencias más altas de cualquier trastorno que las mujeres (30.4\% contra $27 \%$ ). Los trastornos afectivos y de ansiedad son más frecuentes para las mujeres, mientras que los trastornos por uso de sustancias son más frecuentes para los hombres. Acorde a los datos obtenidos en la encuesta nacional de enfermedades psiquiátricas la distribución por estado presenta diferencias, siendo las del estado de Hidalgo, las tasas más elevadas a nivel nacional de depresión en mujeres, seguido por el estado de Jalisco y estado de México. Por su parte los estados de Jalisco y Sonora representan los índices de prevalencia más bajos (Medina et al, 2003). Cabe destacar al respecto, que en el caso de la depresión en los hombres, teóricamente (Bonino, 1998) y algunos estudios (Fleiz, 2010; Sánchez, 2015) consideran la presencia de un subregistro de esa psicopatología en varones debido a la variable género, donde los propios mandatos sociales de actividad, fuerza, rol de proveedor y negación de vulnerabilidad para los varones, no permiten la expresión de los síntomas pertenecientes a los criterios diagnósticos tradicionales de la depresión pasividad, tristeza, llanto incontrolable, dormir en demasía, etc.-, y que se asocian culturalmente con "lo femenino"; en ese sentido, se reflexiona críticamente sobre la posible existencia de una "depresión masculina" con sintomatologías diferenciadas que permiten considera dos tipos: uno activo y otro pasivo, de una psicopatología masculina invisibilizada por la "normalidad" de género (Bonino, 1998).
Dentro de las principales debilidades en materia de atención de salud mental en México encontramos que, aunque un número creciente de la población padece algunos de estos trastornos, solo un bajo porcentaje recibe atención especializada, y generalmente son más mujeres quienes acuden a los servicios. Asimismo, la atención descansa en los grandes hospitales, en consecuencia, los costos de atención resultan elevados. En el caso del estado de Hidalgo, solo se cuenta con un hospital público de atención psiquiátrica, los diagnósticos que más se realizan dentro de este hospital son los trastornos del estado del ánimo y esquizofrenia (IESM-OMS, 2011). Asimismo, los recursos humanos especializados en salud mental -psiquiatras, neuropsicólogos, psicólogos, enfermeros-, que trabajan en el sector público, es reducido y comúnmente sin sensibilidad a la perspectiva de género (Castro y Bronfman, 1993); los servicios que existen están concentrados en las grandes urbes, lo cual dificulta el acceso a las poblaciones rurales e indígenas que suelen vivir en regiones distantes a estas ciudades.

Finalmente, es importante señalar que, existe un escaso contacto entre el sector salud y otros sectores en actividades relacionadas con la salud mental; por ejemplo, sólo un porcentaje bajo de las escuelas públicas cuentan con un psicólogo y en pocas se desarrollan actividades de promoción de la salud y prevención de trastornos mentales.

La Organización Mundial de la Salud -OMS(2011), ha manifestado que las necesidades crecientes de atención en salud mental, requieren programas de intervención creativos y científicamente probados, así como de la voluntad política y consenso social para impulsarlos. Debido a lo anterior, el modelo de Atención en Salud Mental contempla la creación de nuevas estructuras de atención que respeten los derechos de los usuarios, sobre una base sólida dando énfasis en la prevención- la cual se desarrolla principalmente por medio de la difusión de información sobre salud mental y el diagnóstico oportuno que se otorga a los usuarios en los Centros de Salud.

Es importante señalar que las enfermedades mentales también tienen una base biológica (física), ya que, al separar la salud física de la salud mental, da a entender que esta última solo es una cuestión metafísica y dado que sus manifestaciones en su mayoría son a nivel conductual $\mathrm{y} / \mathrm{o}$ emocional se relegan a un 
segundo plano y no se priorizan dentro de las políticas de atención pública. En el caso de las enfermedades mentales, el componente biológico resulta de gran importancia ya que estos trastornos tienen como base alteraciones en la estructura y función del sistema nervioso.

\section{Indicadores de bienestar Hidalgo}

Sobre el contexto particular del estado de Hidalgo, mismo que se debe considerar en el marco de los datos nacionales sobre salud, se analizan algunos datos clave para la comprensión del bienestar relacionado con la salud mental y física de la población del estado. Por ejemplo, la esperanza de vida al nacer 2016 es 74.6 años, siendo la más baja del país para Chiapas con 73.0 y la más alta para el estado de Nuevo león con 76.7 años; respecto de la salud autorreportada en 2014 , la más alta a nivel nacional es para la CDMX con un promedio de 8.5, y la más baja del país con 7.7 para Oaxaca, siendo de 8.1 para Hidalgo; la tasa de obesidad en 2012 para Hidalgo, es de $28.4 \%$, siendo la más alta del país la del estado de Yucatán con 44.8 y la más baja, la de Querétaro con 25.4\%; la tasa de mortalidad materna 2013 para el estado de Hidalgo, indica que por cada 100000 nacidos vivos, se dan 37.5 defunciones de mujeres, siendo las menores cifras las que presenta Nuevo León con una tasa de 14.8, y las mayores, Campeche con 64.5 muertes; sobre la tasa de mortalidad infantil de 2015, las mejores cifras las presenta el estado de Aguascalientes con 8.7 muertes de menores de un año por cada 1000. nacidos, las más altas corresponden al estado de Campeche con 15.5 muertes, y en Hidalgo, la tasa es de 13.1 fallecimientos (INEGI, 2017b).

\section{Los datos para Actopan}

La ciudad de Actopan, Hidalgo está ubicada al norte de la Ciudad de México, de la cual se encuentra a una distancia de $120 \mathrm{~km}$, y a solo 37 $\mathrm{km}$ de la ciudad de Pachuca de Soto, la capital del estado de Hidalgo. Se encuentra dentro de la región geográfica denominada como Valle del Mezquital. De acuerdo con el Censo INEGI, 2010; la localidad tiene una población de 29223 habitantes. Ocupa el séptimo lugar dentro del conteo de las áreas urbanas del estado de Hidalgo. De la población total 13720 son hombres $-46.95 \%$ - y 15503 son mujeres $-53.05 \%$-. De acuerdo a proyecciones del Consejo Nacional de Población, la población en 2015 fue de 30537 habitantes y para 2017 es de 30890 habitantes (SEDESOL, 2017c).
El Municipio colinda al norte con los municipios de Santiago de Anaya y Metztitlán; al este con los municipios de Metztitlán, Atotonilco el Grande, Mineral del Chico y El Arenal; al sur con los municipios de El Arenal, San Agustín Tlaxiaca y Ajacuba; al oeste con los municipios de Ajacuba, San Salvador y Santiago de Anaya (INAFED, 2017).

En 2015, el municipio de Actopan ocupó el lugar 21 de 84 municipios en la escala estatal de rezago social. La población analfabeta es del $4.07 \%$ y la población con educación básica incompleta es de $30.16 \%$. La infraestructura educativa en cuanto a educación básica registra veintiocho preescolares; veintitrés primarias $y$ once secundarias. En educación media superior se cuenta con ocho planteles y en educación superior se cuenta con dos planteles. Cuenta además con una escuela de educación especial, un centro psicopedagógico y aula de apoyo (SEP, 2017). En relación al acceso a los servicios de salud, el $42.06 \%$ de la población no cuenta con derechos para servicios de salud (SSH, 2017). Es decir, las principales problemáticas del municipio están relacionadas con el rezago social y falta de acceso a servicios público de salud y educación.

\section{Comunidad de Dhaxtá}

Se encuentra en la región geográfica del estado de Hidalgo denominada Valle del Mezquital. La localidad se localiza en la región oriente de México, al centro del estado de Hidalgo y al oeste del territorio municipal de Actopan (SEDESOL, 2017a). Cuenta con una población de 2,313 habitantes, de los cuales 1,101 son hombres y 1,212 son mujeres. Esta población corresponde al $4.26 \%$ de la población municipal y es considerada una comunidad rural.

El $41.94 \%$ de la población no cuenta con derecho a servicios de salud. El grado de marginación se considera como medio y el grado de rezago social para la localidad se considera muy bajo (SEDESOL, 2017b). El $5.57 \%$ de la población de 15 años o más es analfabeta, el $2.97 \%$ de la población de 6 a 14 años que no asiste a la escuela y el $41.74 \%$ de la población de 15 años y más tiene educación básica incompleta. La localidad cuenta con tres escuelas de nivel preescolar, dos primarias, una telesecundaria, tres bachilleratos y una universidad (SEP,2017). Las principales actividades económicas son el comercio, la agricultura, ganadería y la avicultura. 


\section{Promoción de la salud}

Para la Organización Mundial de la Salud -OMS-, promover la salud consiste en capacitar a las personas, las comunidades y las sociedades, y dotarlas de medios para que se ocupen de su salud y su calidad de vida (OMS, 2017). ¿Cómo pueden todos los habitantes del planeta, vivan donde vivan - en ciudades o en aldeas remotas lograr el más alto nivel de salud? Para ello, es crucial poner en marcha medidas pangubernamentales, disponer de entornos sanos y propiciar un público informado y capaz de tomar decisiones propias.

Desde este enfoque, se considera que una población sana es también fundamental en el progreso hacia la Agenda 2030 para el Desarrollo Sostenible $^{3}$. La Novena Conferencia Mundial de Promoción de la Salud, que se celebró del 21 al 24 de noviembre de 2016 en Shanghai, se centró en estas y otras acciones innovadoras para promover la salud (OMS, 2017). La promoción de la salud permite que las personas tengan un mayor control de su propia salud. Abarca una amplia gama de intervenciones sociales $y$ ambientales destinadas a beneficiar y proteger la salud y la calidad de vida individuales, mediante la prevención y solución de las causas primordiales de los problemas de salud, y no centrándose únicamente en el tratamiento y la curación. La promoción de la salud tiene tres componentes esenciales (OMS, 2016a):

\section{Buena gobernanza sanitaria}

La promoción de la salud requiere que los formuladores de políticas de todos los departamentos gubernamentales hagan de la salud un aspecto central de su política. Esto significa que deben tener en cuenta las repercusiones sanitarias en todas sus decisiones, y dar prioridad a las políticas que eviten que la gente enferme o se lesione.

Estas políticas deben ser respaldadas por regulaciones que combinen los incentivos del sector privado con los objetivos de la salud pública, por ejemplo, armonizando las políticas fiscales que gravan los productos nocivos 0 insalubres, como el alcohol, el tabaco y los alimentos ricos en sal, azúcares o grasas, con medidas para estimular el comercio en otras áreas. Asimismo, hay que promulgar leyes que respalden la urbanización saludable mediante la facilitación de los desplazamientos a pie, la reducción de la contaminación del aire y del agua 0 el cumplimiento de la obligatoriedad del uso del casco y del cinturón de seguridad.

\section{Educación sanitaria}

Las personas han de adquirir conocimientos, aptitudes e información que les permitan elegir opciones saludables, por ejemplo, con respecto a su alimentación y a los servicios de salud que necesitan. Tienen que tener la oportunidad de elegir estas opciones y gozar de un entorno en el que puedan demandar nuevas medidas normativas que sigan mejorando su salud.

\section{Ciudades saludables}

Las ciudades tienen un papel principal en la promoción de la buena salud. El liderazgo y el compromiso en el ámbito municipal son esenciales para una planificación urbana saludable y para poner en práctica medidas preventivas en las comunidades y en los centros de atención primaria. Las ciudades saludables contribuyen a crear países saludables y, en última instancia, un mundo más saludable.

A partir de estos tres componentes esenciales de la promoción de la salud, en la declaración de Shangai (OMS, 2016b), se deslindan varios ejes que involucran a diferentes actores públicos y sociales desde el reconocimiento de que la salud y el bienestar son fundamentales para el desarrollo sostenible. De esta forma, se considera a las autoridades de los niveles estatal y local, desde el entendimiento de que la buena gobernanza es esencial para la salud, y se realiza un "Llamamiento a la acción", donde se reconoce que la salud es una opción política, y que se deben contrarrestar los intereses perjudiciales para la salud y eliminar los obstáculos al empoderamiento de las poblaciones en condiciones más vulnerables; se insta a los líderes políticos de diferentes sectores y niveles de gobernanza, del sector privado y de la sociedad civil, a unirse en la determinación de promover la salud y el bienestar en todos los Objetivos del Desarrollo Sostenible -ODS-; además, se debe considerar que la promoción de la salud requiere una acción coordinada por todas las partes interesadas, ya que es una responsabilidad compartida. 
Con base en la buena gobernanza, el papel de actores sociales y la comunidad, se identifican ciertos ejes de trabajo que implicarían de forma más directa el involucramiento de la comunidad en la realización de los objetivos de esta agenda internacional planteada por la OMS, siendo los siguientes:

Cuando se considera promover la salud mediante la adopción de medidas encaminadas al cumplimiento de todos los ODS, implica la necesidad de adoptar medidas de manera decidida respecto de todos los determinantes sociales de la salud -DSS-, empoderar a las personas para que tengan un mayor control sobre su salud y velar por que los sistemas de salud estén centrados en las personas. En este sentido, la identificación y problematización de los DSS mediante el diagnóstico y la investigación, así como el empoderamiento de las personas vía la información y sobre sus opciones y derecho a la salud, resultan acciones estratégicas para las comunidades.

Adoptar decisiones políticas audaces en pro de la salud para "no dejar a nadie atrás", conlleva la adopción de medidas decididas a favor de los derechos de las mujeres, las poblaciones que se desplazan y del creciente número de personas afectadas por crisis humanitarias y ambientales. En este sentido, la buena gobernanza resulta prioritaria, así como la acción local a través de las ciudades y comunidades, y el empoderamiento de las personas mediante la promoción de los conocimientos sanitarios. Este eje también concede una alta prioridad a la innovación y al desarrollo para contribuir a que las personas disfruten de una vida sana, y a la salud de los más vulnerables. La articulación de las tareas comunitarias con las decisiones políticas en pro de la salud, mediante la promoción de los conocimientos sanitarios puede resultar clave para la prevención de la salud de poblaciones consideradas vulnerables.

Las ciudades y las comunidades son entornos esenciales para la salud, por lo tanto, la salud es el producto de la vida diaria, esto es, de los barrios y las comunidades donde la gente convive y realiza sus diferentes actividades. Para la OMS, la salud es uno de los indicadores más eficaces de que una ciudad está experimentando un desarrollo sostenible, y contribuye a que las ciudades sean lugares incluyentes, seguros y resilientes para el conjunto de la población. Junto con los dirigentes de las ciudades se debe abordar la combinación tóxica de la rápida migración del campo a la ciudad, los desplazamientos mundiales de población, el estancamiento económico, el elevado desempleo y la pobreza, así como el deterioro del medio ambiente y la contaminación. Se considera inaceptable que los habitantes de las ciudades de zonas pobres padezcan de forma desproporcionada problemas de salud y tengan dificultades para acceder a los servicios sanitarios. Por lo tanto, se busca:

aplicar plenamente los mecanismos de que disponen los gobiernos para proteger la salud y promover el bienestar mediante políticas públicas; reforzar la legislación, la reglamentación y la fiscalidad de los productos no saludables;

dar prioridad a las políticas que generen beneficios conjuntos para la salud y el bienestar y a las políticas urbanas en otros ámbitos, aprovechando al máximo las tecnologías de innovación e interacción sociales;

ayudar a las ciudades a promover la equidad y la inclusión social, aprovechando los conocimientos, las capacidades y las prioridades de sus diferentes poblaciones mediante un fuerte compromiso comunitario;

reorientar los servicios sanitarios y sociales para optimizar el acceso equitativo y dar a las personas y las comunidades un papel preponderante.

Finalmente, se considera que los conocimientos sanitarios empoderan y fomentan la equidad: empoderan a las personas y posibilitan su participación en iniciativas colectivas de promoción de la salud. El hecho de que los encargados de la adopción de decisiones y los inversores tengan un alto nivel de conocimientos sanitarios contribuye a que se impliquen más en favor de los resultados sanitarios, los beneficios conjuntos y las intervenciones eficaces respecto de los determinantes de la salud. Los conocimientos sanitarios se basan en un acceso incluyente y equitativo a una educación de calidad y al aprendizaje permanente. Debe ser una parte integrante de las capacidades y competencias desarrollas a lo largo de la vida, primera y principalmente a través de los planes de estudio escolares. De esta forma, se plantean las siguientes acciones:

reconocer los conocimientos sanitarios como un determinante fundamental de la salud y a invertir en su desarrollo;

formular, aplicar y hacer un seguimiento de las estrategias intersectoriales nacionales y locales para reforzar los conocimientos sanitarios de 
todas las poblaciones y en todos los entornos educativos;

aumentar el control de los ciudadanos sobre su propia salud y sus determinantes mediante el aprovechamiento del potencial de la tecnología digital;

Las escuelas de educación superior juegan un papel importante en la acciones de promoción de la salud, ya que estas acciones pueden ser compatibles con las tareas universitarias, por ejemplo, difundir conocimientos sanitarios estratégicos para impactar en ciertas DSS; potencializar el impacto de las acciones públicas de promoción de la salud mediante el fortalecimiento de las estrategias implementadas en la comunidad por parte de los sistemas de salud y de desarrollo social; promover las elecciones saludables en el consumo para favorecer una mejor salud, y; usar la tecnología para facilitar todas las tareas anteriores $y$ desarrollar nuevas alternativas de promoción de la salud. En este sentido, la comunidad de Dhaxtá cuenta con una ventaja ya que dentro de esta se encuentran las instalaciones de la Escuela Superior de Actopan, perteneciente a la Universidad Autónoma del Estado de Hidalgo.

En síntesis, a partir de los ejes planteados en la Declaración de Shangai sobre Promoción de la Salud (OMS, 2016b), se pueden implementar las siguientes acciones estratégicas:

Identificar y problematizar de los DSS que afectan a la comunidad, mediante el diagnóstico y la investigación;

difundir conocimientos sanitarios estratégicos para impactar en la disminución de las DSS;

promover elecciones saludables en el consumo para favorecer una mejor salud;

la promoción de conocimientos sanitarios clave para la prevención de la salud, en las poblaciones de la comunidad consideradas vulnerables;

\section{Referencias Bibliográficas}

Arredondo, J., Carranza, N., Vázquez, M. y Rodríguez, M. (2003). Transición epidemiológica. Acta pediátrica de México, Vol. 24, núm. 1, 46-56; enero- febrero 2003. Disponible en: http://www.imbiomed.com.mx/1/1/articulos.php?method=showDetail \&id_articulo=13218\&id_seccion=96\&id_ejemplar=1355\&id_revista= 17 promover la equidad y la inclusión social mediante la optimización de los recursos y conocimientos comunitarios;

el empoderamiento de las personas de la comunidad sobre sus opciones para acceder a sistemas de salud y su derecho a la salud;

fortalecer las estrategias implementadas en la comunidad por parte de los sistemas de salud y de desarrollo social;

desarrollar estrategias adecuadas para la canalización de las necesidades de salud de las poblaciones, hacia las instancias públicas y civiles pertinentes para su atención;

usar la tecnología para facilitar todas las tareas anteriores y desarrollar nuevas estrategias para la promoción de la salud.

El panorama presentado sobre las principales problemáticas de salud en la comunidad de EI Dhaxtá, muestran un patrón similar al perfil del municipio de Actopan, y en congruencia con las del estado de Hidalgo y del territorio nacional, con la presencia simultánea de enfermedades infectocontagiosas $y$, en segundo término, las crónico-degenerativas. Dicho patrón reflejaría, por un lado, altos índices de pobreza, deficiencias en la calidad de vida de la población y otras determinantes sociales de la salud, y por otro, el efecto de los avances científicos en la atención de la salud. Así mismo, se identifican diferenciales en salud producto del género y la edad, mostrando patrones específicos de morbilidad y mortalidad en los datos a nivel nacional, estatal y municipal. Finalmente, considerando las emergentes problemáticas nacionales de salud, se señalan líneas estratégicas de atención a la salud física y mental desde un enfoque de promoción de la salud, dirigidas a sentar las bases para la implementación de acciones que se reflejen en una mejora de las condiciones de salud de la comunidad de Daxthá, mismas que se encuentran en la fase de diseño y gestión comunitaria por parte de la universidad de adscripción de los autores de este trabajo.
Bonino, L. (1998). Deconstruyendo la 'Normalidad' Masculina: Apuntes para una 'psicopatología' de género masculino. Actualidad Psicológica, 253.

Castro R. y Bronfman M. (1993). Teoría Feminista y Sociología Médica: bases para una discusión. Cad. Saúde Públ., 9 (3): jul/set. 
CONEVAL, (2012), Consejo Nacional de Evaluación de la Política de Desarrollo Social. Medición de la Pobreza, Gobierno de México. Recuperado de http://www.coneval.gob.mx/Medicion/Paginas/Medici\%c3\%b3n/Pobre za\%20 2012/Pobreza-2012.aspx

CONEVAL, (2015), Consejo Nacional de Evaluación de la Política de Desarrollo Social. Medición de la Pobreza, 2015. Gobierno de México. Recuperado de

http://www.coneval.gob.mx/Medicion/Documents/Pobreza\%202014_ CONE VAL_web.pdf

Connell, R. (2003). Masculinidades. México: PUEG/UNAM. de Keijzer, B. (2014). Hombres, género y políticas de salud en México. En Figueroa,J.A. (Ed.), Políticas públicas y la experiencia de ser hombre. México: Centro de Estudios Demográficos, Urbanos y Ambientales del El Colegio de México.

Fleiz, C. (2010). El malestar depresivo: una experiencia vivida por los hombres a través de su construcción de género (Tesis inédita de doctorado en Psicología). Psicología social y ambiental, Universidad Nacional Autónoma de México.

Guerrero, L. y León, A. (2010). Estilo de vida y salud. Educere, vol. 14, núm. 48, enero-junio, 2010, pp. 13-19 Universidad de los Andes Mérida, Venezuela. Disponible en: http://www.redalyc.org/pdf/356/35616720002.pdf

IESM-OMS, (2011), Informe sobre sistema de salud mental en México, México: Secretaria de Salud, Organización Panamericana de la Salud, Organización Mundial de la Salud, p 58.

INAFED, (2017), Instituto Nacional para el federalismo y el desarrollo Municipal, «Regionalización del estado de Hidalgo». Secretaría de Gobernación. Consultado el 14 de diciembre de 2017. http://www.inafed.gob.mx/work/enciclopedia/EMM13hidalgo/regional izacion.html

INEGI, (2012), Instituto Nacional de Estadística y Geografía. Encuesta Nacional de Ingresos y Gastos de los Hogares, 2012. Principales resultados. Recuperado de

http://www3.inegi.org.mx/sistemas/microdatos/microdatos_archivos/e nigh/d oc/resutados_enigh12.pdf

INEGI, (2015), Encuesta Nacional de Ocupación y Empleo. Indicadores de Ocupación y Empleo. Cifras oportunas durante enero de 2015. Instituto Nacional de Estadística y Geografía. Recuperado de http://www.inegi.org.mx/saladeprensa/boletines/2015/iooe/iooe2015_0 2.pdf

INEGI, (2017a), Estadísticas a propósito del día de muertos" (2 de noviembre). Datos nacionales. Recuperado de http://www.inegi.org.mx/saladeprensa/aproposito/2017/muertos2017 Nal.pdf

INEGI, (2017b), Indicadores de bienestar por entidad federativa. Instituto de Estadística y Geografía. Consultado el 19 de diciembre de 2017. http://www.beta.inegi.org.mx/app/bienestar/?ag=13\#grafica

INEGI, (2017c), Actopan, Hidalgo, Prontuario de información geográfica municipal de los Estados Unidos Mexicanos. Consultado el 14 de diciembre de 2017.

http://www3.inegi.org.mx/contenidos/app/mexicocifras/datos_geografi $\cos / 13 / 13003 . p d f$

López, O., Blanco, J., Garduño, M. A., Granados, J. A., Jarillo, E., López, S., Rivera,

J.A. \& Tetelboin, C. (2010). Los determinantes sociales de la salud y la acción política. Una perspectiva desde la academia. En Asociación
Latinoamericana de Medicina Social, Taller Latinoamericano sobre determinantes de la Salud. Uruguay: ALAMES.

López O. y Blanco J. (2003). Desigualdad social e inequidades en salud. Desarrollo de conceptos y comprensión de relaciones. Salud Problema, Nueva Época, 8 (14-15): 7-16.

Medina-Mora, Ma. E, et al. (2003). Prevalencia de trastornos mentales y uso de servicios: Resultados de la encuesta Nacional de Epidemiología Psiquiátrica, Salud mental, 26(4), 1-16.

OMS, (2015), Salud Mental, Consultado el 19 de diciembre de 2017. http://www.who.int/topics/mental_health/es/

OMS, (2016a), ¿Qué es la promoción de la salud? Organización Mundial de la Salud. Consultado el 19 de diciembre de 2017.http://www.who.int/features/qa/health-promotion/es/

OMS, (2016b), Declaración de Shangai sobre la Promoción de la Salud en la Agenda 2030 para el Desarrollo Sostenible. $9^{\mathrm{a}}$ Conferencia mundial de Promoción de la Salud; Shangai, 21-24 de noviembre de 2016. http://www.who.int/healthpromotion/conferences/9gchp/Shanghaideclaration-final-draft-es.pdf.pdf

OMS, (2017) Promover la salud, promover el desarrollo sostenible. Organización Mundial de la Salud, Consultado el 19 de diciembre de 2017. http://www.who.int/healthpromotion/es/

Sánchez, J. (2015). Narraciones sobre la Experiencia Depresiva de Hombres: Masculinidad y Narcisismo. (Tesis inédita de doctorado). Programa de Maestría y Doctorado en Psicología y Salud, de la Universidad Nacional Autónoma de México.

Scott, J. (1990). El género como una categoría útil para el análisis histórico. En Amelang J. \& Nash, M. (Eds.), Historia y género: las mujeres en la Europa moderna y contemporánea. España: Universidad de Valencia.

SEDESOL, (2017a) Cédulas de Información Municipal. «Municipio de Actopan, Hidalgo». Secretaría de Desarrollo Social. Gobierno de México. Consultado el 14 de diciembre de 2017.

http://www.microrregiones.gob.mx/zap/datGenerales.aspx?entra=nacion\& ent $=13 \&$ mun $=003$

SEDESOL, (2017b) Catálogo Localidades - Microrregiones. «Localidad de El Daxtha, Actopan, Hidalgo». Secretaría de Desarrollo Social Gobierno de México. Consultado el 15 de diciembre: http://www.microrregiones.gob.mx/catloc/contenido.aspx?refnac=130 030012

SEP, (2017), Directorio de escuelas. Sistema Nacional de Información de Escuelas. Consultado el 14 de diciembre de 2017. http://www.snie.sep.gob.mx/SNIESC/

SIDSS (2015), Informe sobre la salud de los mexicanos, 2015. Subsecretaría de Integración y Desarrollo del Sector Salud Dirección General de Evaluación del Desempeño. Gobierno de México.

Recuperado de https://www.gob.mx/salud/documentos/informesobre-la-salud-de-los- mexicanos-2015

SSH, (2017), Secretaria de Salud del Estado de Hidalgo, Actualización del Programa. Sectorial de Salud 2011-2016. Gobierno del estado de Hidalgo. Consultado el 14 de diciembre de 2017.

http://siieh.hidalgo.gob.mx/Programas\%20Sectoriales/files/programa_sect orial_sal ud.pdf 


\section{Diez principales causas de morbilidad por sexo}

\begin{tabular}{|c|c|c|c|c|}
\hline \multirow[b]{2}{*}{ Posición } & \multicolumn{2}{|l|}{ Hombres } & \multicolumn{2}{|l|}{ Mujeres } \\
\hline & Padecimiento & Casos & Padecimiento & Casos \\
\hline 1 & $\begin{array}{l}\text { Infecciones respiratorias } \\
\text { agudas }\end{array}$ & $12,120,993$ & $\begin{array}{l}\text { Infecciones respiratorias } \\
\text { agudas }\end{array}$ & $15,372,246$ \\
\hline 2 & Infecciones Intestinales & $2,272,667$ & Infecciones Intestinales & $3,246,261$ \\
\hline 3 & Infección de vía urinarias & 997,792 & $\begin{array}{l}\text { Infección de vía } \\
\text { urinarias }\end{array}$ & $2,668,760$ \\
\hline 4 & Ulceras, gastritis y duodenitis & 529,636 & $\begin{array}{l}\text { Ulceras, gastritis y } \\
\text { duodenitis }\end{array}$ & 968,166 \\
\hline 5 & $\begin{array}{l}\text { Gingivitis y enfermedad } \\
\text { periodontal }\end{array}$ & 415,435 & $\begin{array}{l}\text { Gingivitis y enfermedad } \\
\text { periodontal }\end{array}$ & 703,724 \\
\hline 6 & Otitis media aguda & 272,191 & Otitis media aguda & 367,434 \\
\hline 7 & Conjuntivitis macro purulenta & 201,215 & Vulvovaginitis aguda & 355,600 \\
\hline 8 & Hipertensión arterial & 194,258 & Hipertensión arterial & 286,074 \\
\hline 9 & Diabetes mellitus & 157,793 & Conjuntivitis & 255,068 \\
\hline 10 & $\begin{array}{l}\text { Intoxicación por picaduras de } \\
\text { alacrán }\end{array}$ & 146,330 & Candidiasis urogenital & 235,372 \\
\hline
\end{tabular}

Tabla2 Diez principales causas de morbilidad por grupo de edad

\begin{tabular}{|c|c|c|c|c|c|c|c|c|c|c|c|}
\hline \multirow[t]{2}{*}{ Padecimiento } & \multicolumn{11}{|c|}{ Grupos de edad } \\
\hline & $<1$ & $\begin{array}{l}1- \\
4\end{array}$ & $\begin{array}{l}5- \\
9\end{array}$ & $\begin{array}{l}10- \\
14\end{array}$ & $\begin{array}{l}15- \\
19\end{array}$ & $\begin{array}{l}20- \\
24\end{array}$ & $\begin{array}{c}25- \\
44\end{array}$ & $\begin{array}{r}45- \\
49\end{array}$ & $\begin{array}{l}50- \\
59\end{array}$ & $\begin{array}{l}60- \\
64\end{array}$ & $\begin{array}{l}65 \\
>\end{array}$ \\
\hline respiratorias & 1 & 1 & 1 & 1 & 1 & 1 & 1 & 1 & 1 & 1 & 1 \\
\hline Infecciones Intestinales & 2 & 2 & 2 & 2 & 3 & 3 & 3 & 3 & 3 & 3 & 3 \\
\hline Infección de vía urinarias & 4 & 3 & 3 & 3 & 2 & 2 & 2 & 2 & 2 & 2 & 2 \\
\hline Ulceras, gastritis y duodenitis & 16 & 16 & 16 & 4 & 4 & 4 & 4 & 4 & 4 & 4 & 4 \\
\hline $\begin{array}{l}\text { Gingivitis y enfermedad } \\
\text { periodontal }\end{array}$ & 15 & 14 & 7 & 5 & 5 & 5 & 5 & 5 & 5 & 5 & 6 \\
\hline Otitis media aguda & 6 & 4 & 4 & 6 & 6 & 7 & 8 & 9 & 9 & 10 & 10 \\
\hline Hipertensión arterial & 18 & 18 & 18 & 20 & 19 & 18 & 10 & 6 & 6 & 6 & 5 \\
\hline Conjuntivitis & 3 & 6 & 8 & 7 & 9 & 10 & 11 & 11 & 10 & 9 & 9 \\
\hline Diabetes mellitus & 18 & 18 & 18 & 19 & 20 & 20 & 13 & 7 & 7 & 7 & 7 \\
\hline Obesidad & 12 & 15 & 14 & 14 & 13 & 11 & 7 & 8 & 8 & 8 & 11 \\
\hline Vulvovaginitis aguda & 17 & 17 & 17 & 16 & 7 & 6 & 6 & 10 & 11 & 15 & 17 \\
\hline Asma & 10 & 7 & 6 & 8 & 14 & 14 & 16 & 14 & 13 & 13 & 14 \\
\hline $\begin{array}{l}\text { Intoxicación por picadura de } \\
\text { alacrán }\end{array}$ & 14 & 13 & 11 & 9 & 8 & 9 & 12 & 13 & 12 & 12 & 12 \\
\hline Amebiasis intestinal & 8 & 8 & 9 & 10 & 11 & 13 & 15 & 15 & 14 & 14 & 13 \\
\hline Candidiasis urogenital & 18 & 18 & 18 & 18 & 10 & 8 & 9 & 12 & 16 & 18 & 19 \\
\hline Síndrome febril & 9 & 10 & 12 & 13 & 12 & 12 & 14 & 16 & 17 & 17 & 16 \\
\hline Varicela & 7 & 5 & 5 & 12 & 17 & 17 & 20 & 20 & 20 & 20 & 20 \\
\hline Otras helmintiasis & 13 & 9 & 10 & 11 & 15 & 15 & 17 & 17 & 15 & 16 & 15 \\
\hline Neumonía y bronconeumonía & 5 & 11 & 15 & 17 & 18 & 19 & 19 & 19 & 18 & 11 & 8 \\
\hline
\end{tabular}


Tabla 3.Principales causas de mortalidad en México.

Causas de defunción
- Agresiones

- Accidentes de transporte

- Lesiones auto infligidas

- Otras totaEnfermedades depríngipales

- Otras causas

\section{Enfermedades del sistema circulatorio}

- Enfermedades Isquémicas del corazón

- Enfermedades cerebrovasculares

- Enfermedades Hipertensivas

- Otras

Enfermedades Endócrinas, nutricionales metabólicas

- Diabetes mellitus

- Desnutrición

- Otras

Tumores (neoplasias)

- De la tráquea, pulmón

- De la próstata

- Del hígado

- De la mama

- Del Estomago

- Otros

Causas externas de morbilidad y mortalidad

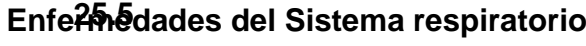

13.4Enfermedades crónicas

-5.2 Neumonía 4

.3.5 Otras

6

3.4

17.5

Otras Causas

Totah 5.0

1

13.0

1.0

1.0

1.0

1.0

0.9

8.1

10.4 